\title{
Unlocking the potential of retinoic acid in anticancer therapy
}

\author{
T Schenk ${ }^{1,3}$, S Stengel ${ }^{1,3}$ and A Zelent ${ }^{\star, 2}$ \\ ${ }^{1}$ Haemato-Oncology Research Unit, Division of Molecular Pathology, The Institute of Cancer Research, 123 Old Brompton Road, \\ SW7 3RP London, UK and 'Division of Hemato-Oncology, Department of Medicine, Miller School of Medicine, Sylvester \\ Comprehensive Cancer Center, 1550 NW 10th Avenue, Miami, FL 33136, USA
}

\begin{abstract}
All-trans-retinoic acid (ATRA) is a physiologically active metabolite of vitamin A. Its antitumour activities have been extensively studied in a variety of model systems and clinical trials; however, to date the only malignancy responsive to ATRA treatment is acute promyelocytic leukaemia (APL) where it induces complete remission in the majority of cases when administered in combination with light chemotherapy and/or arsenic trioxide. After decades of studies, the efficacy of ATRA to treat other acute myeloid leukaemia (AML) subtypes and solid tumours remains poor. Recent studies directed to improve ATRA responsiveness in non-APL AML seem to indicate that the lack of effective ATRA response in these tumours may be primarily due to aberrant epigenetics, which negatively affect ATRA-regulated gene expression and its antileukaemic activity. Epigenetic reprogramming could potentially restore therapeutic effects of ATRA in all AML subtypes. This review discusses the current progresses in the understanding how ATRA can be utilised in the therapy of non-APL AML and other cancers.
\end{abstract}

Shortly after the discovery of vitamin A (retinol) in 1913, scientists began to realise the extreme importance of this simple fat-soluble molecule in a large number of complex and diverse physiological processes in vertebrates. All-trans-retinoic acid (ATRA) was identified as a key physiologically active metabolite of vitamin A. The understanding of how ATRA exerts its highly pleiotropic effects in vertebrates, as well as its antitumour activities, remained unclear until the discovery of nuclear retinoic acid receptors. Retinoic acid receptors were shown to be members of the superfamily of steroid/thyroid hormone nuclear receptors functioning primarily as ligand-inducible transcription factors.

\section{RETINOIC ACID RECEPTORS}

Similarly to other key developmental regulators, RARs possess the capacity to function as a molecular switch; when not bound by ligand, they form a complex with co-repressors such as $\mathrm{N}$-CoR (negative co-regulator) or SMRT (silencing mediator for retinoid and thyroid hormone receptors), and histone deacetylases (HDACs) to actively inhibit gene expression (Figure 1). Upon ATRA binding, co-repressors are released and co-activators, including histone acetyltransferases (HATs), DRIP/TRAP/ARC, or mediator-containing complexes are recruited to decompress chromatin and activate transcription of target genes (Glass and Rosenfeld, 2000). To date, three different RAR and retinoid $X$ (or rexinoid) receptor $(R X R)$ genes have been characterised $(R A R A$, $R A R B$, and $R A R G$ ), each encoding multiple $\mathrm{N}$-terminal protein isoforms. Retinoid X (or rexinoid) receptors, which bind 9-cisretinoic acid (9-cis-RA) with high affinity, serve as obligatory heterodimerisation partners for RARs. RARA, in common with other $R A R$ genes, encodes two major isoforms (Figure 1) that differ in their $\mathrm{A}$ region sequence that contribute to transcriptional regulation in a ligand-independent and promoter-specific manner (Leid et al, 1992). These isoforms are identical in their B to $\mathrm{F}$ region sequence, which contain DNA (DBD) and ligand binding (LBD) domains as well as structural motifs responsible for dimerisation, co-repressor interaction, and ligand-dependent trans-activation. Expression of the $\mathrm{RAR} \alpha 2, \mathrm{RAR} \beta 2$ and possibly also $\operatorname{RAR} \gamma 2$ isoforms is under control of promoters that are inducible by ATRA. Activation of transcription by RARs is intrinsically linked to their proteasome-mediated degradation (Giannì et al, 2002) and upregulation of RAR $\alpha 2, \operatorname{RAR} \beta 2$, and $\mathrm{RAR} \gamma 2$ isoform expression by ATRA may therefore have been evolutionarily selected and conserved to renew expression of a

${ }^{*}$ Correspondence: Dr A Zelent; E-mail: a.zelent@med.miami.edu

${ }^{3}$ These authors contributed equally to this work. 


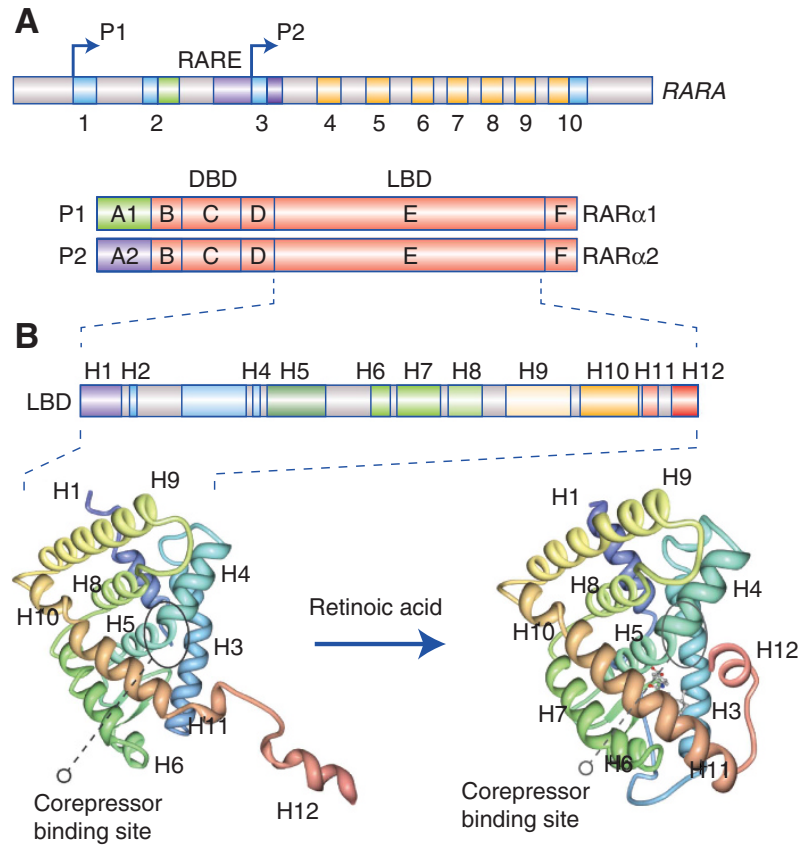

C

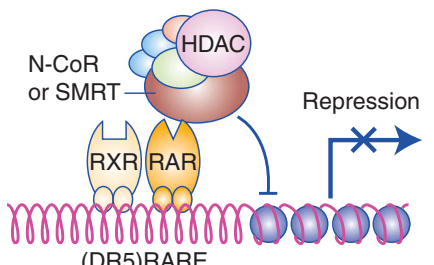

(DR5)RARE

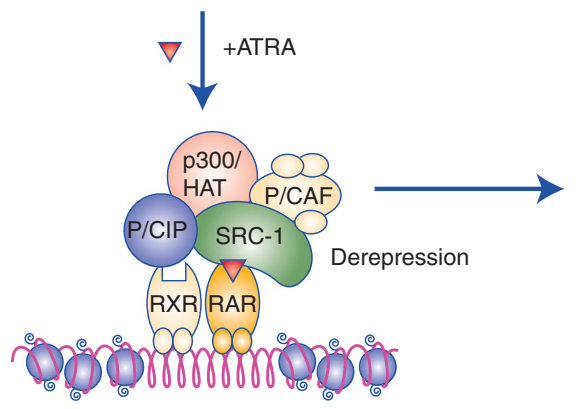

(DR5)RARE

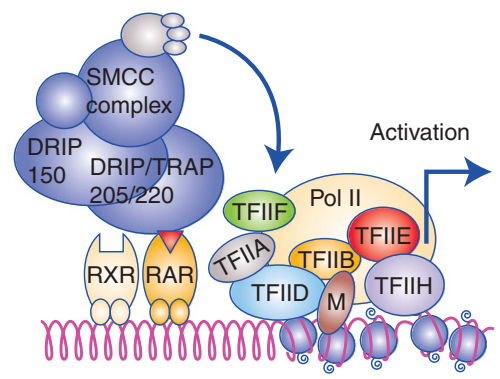

(DR5)RARE

Figure 1. Structure and function of the retinoic acid receptor alpha gene and protein upon binding of RA. (A) RARA, in common with other RAR genes, encodes two major isoforms that differ in their promoters (P1 and P2) and A region sequences, but are identical in their B-F region sequences. The B-F regions contain DNA (DBD) and ligand binding (LBD) domains as well as structural motifs responsible for dimerisation, co-repressor interaction and ligand-dependent trans-activation. (B) Schematic representation of the RAR $\alpha$ LBD, which is composed of 12 alpha helices (colour coded and labelled as $\mathrm{H} 1-\mathrm{H} 12$ ). The ribbon diagrams of the crystal structures of un-liganded RXR $\mathrm{LBD}$ (3A9E) and the liganded RAR $\alpha$ LBD (3KMR) are illustrating the principle of RAR activation. In the un-liganded state, helices $\mathrm{H} 3$ and $\mathrm{H} 4$ provide a binding site for co-repressors. Upon ligand binding $\mathrm{H} 3$ and $\mathrm{H} 4$ undergo conformational changes leading to a destruction of the co-repressor binding site. Helix H12 moves towards a ligand binding pocket and generates a defined binding surface for co-activators such as p160. (C) RARs possess the capacity to function as a molecular switch; when not bound by ligand, they complex with co-repressors, such as N-CoR or SMRT, and HDACs to actively inhibit gene expression. Upon ATRA binding, co-repressors are released and co-activators, including DRIP/TRAP/ARC or mediator-containing complexes as well as HATs, are recruited to decompress chromatin and activate transcription of target genes.

given receptor to sustain gene activation and the physiological effects of ATRA over an extended period of time.

\section{NON-GENOMIC EFFECTS OF ATRA}

Similar to other nuclear receptor ligands ATRA can also function, via so-called non-genomic pathways, independent of the classical mechanism of nuclear receptor action. All-trans-retinoic acid can effectively and rapidly modulate the activities of proteins involved in signal transduction in a manner that is highly cell type specific. The activation of multiple kinase signalling pathways leads to transcriptional effects independent of nucleolar RARs via transcription factors that lie at the end of these signalling cascades (Al Tanoury et al, 2013; see Figure 2). For example, in neuronal cells, ATRA has been shown to activate Erks via PI3K and Src kinases leading to repression of the antidifferentiative 


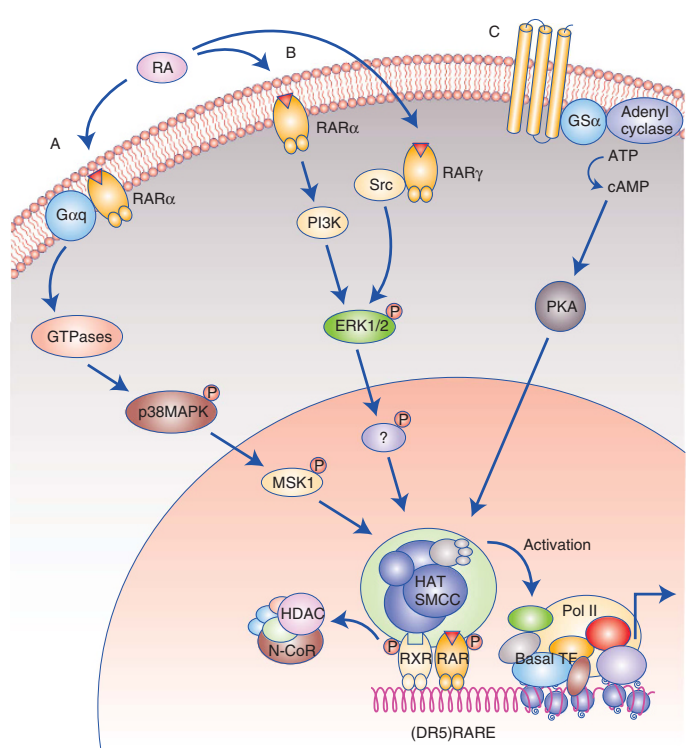

Figure 2. Non-genomic effects of ATRA. (A) A fraction of the cellular RAR $\alpha$ pool is present in membrane lipid rafts, where it forms complexes with $\mathrm{G}$ protein alpha $\mathrm{Q}(\mathrm{G} \alpha q)$ in response to RA. Activation of $\mathrm{G} \alpha \mathrm{q}$ leads to activation of RhoGTPase, P38MAPK and MSK1 in epithelial and fibroblast cells. Phospho-MSK1 contributes to transcriptional activation of RAR $\alpha$ target genes by phosphorylation of histones and RAR $\alpha$ itself. (B) In neuronal cells, RA was shown to activate Erks via PI3K/RAR $\alpha$ and $\mathrm{Src} / \mathrm{RAR} \gamma$. Downstream effectors of Erks are still unknown, but in RAtreated P19 cells, the activated Erks phosphorylate testicular nuclear receptor 2 (TR2) that then becomes a repressor of the octamer-binding transcription factor 4 (Oct4) gene, thus facilitating the differentiation program that is expected to take place in response to RA. (C) RA leads to a rapid response to cyclic adenosine monophosphate (AMP) accumulation in APL cells, most likely by stimulation of adenyl cyclase, leading to protein kinase A (PKA) activation and enhanced expression of RAR target genes and differentiation.

octamer-binding transcription factor 4 (Oct4). In epithelial cells and fibroblasts, $\mathrm{RAR} \alpha$ can form complexes and activate $\mathrm{G}$ protein alpha $\mathrm{Q}(\mathrm{G} \propto \mathrm{q})$ in response to ATRA. Activation of $\mathrm{G} \alpha \mathrm{q}$ in turn leads to the activation of RhoGTPase, p38MAPK, and MSK1. Phospho-MSK1 then contributes to transcriptional activation of $\operatorname{RAR} \alpha$ target genes by phosphorylation of histones and $\mathrm{RAR} \alpha$ itself.

Another non-genomic effect of ATRA is that it can rapidly lead to the accumulation of cyclic adenosine monophosphate (AMP) in acute promyelocytic leukaemia (APL), most likely by adenyl cyclase stimulation and protein kinase A (PKA) activation. Activated PKA can phosphorylate and activate RAR $\alpha$ leading to increased expression of RAR target genes and differentiation. In addition, ATRA has been shown to upregulate the granulocyte colony-stimulating factor (G-CSF) and granulocyte macrophage colony-stimulating factor (GM-CSF) receptor resulting in the amplification of the MAP kinase pathway via a positive feedbackloop mechanism (Glasow et al, 2005). Therefore, in normal myelomonocytic differentiation ATRA and growth factor (GF) signalling converge via common signalling cascades to affect activities of RARs as well as other transcription factors.

The activity of ATRA in leukaemia cells is dependent on complex interactions between genomic and non-genomic pathways. Although much remains to be understood regarding the exact mechanisms underlying the non-genomic effects of ATRA, it is clear that these effects have important consequences and have to be taken into consideration when evaluating the overall response of a particular cell type to retinoids.

\section{ATRA IN APL}

Defective or aberrant differentiation is a hallmark of many cancers. It is caused by various mutations that disrupt signalling pathways, epigenetic modifiers, and transcription factors leading to the deregulation of the expression of proteins required for differentiation. Terminal differentiation results in the loss of long-term proliferative capacity and eventually induction of apoptosis. The potential of ATRA to force the completion of disrupted maturation programs underlies the concept of differentiation therapy. Differentiation therapy offers the prospect of a less aggressive treatment coupled to limited damage to normal cells.

Breitman et al (1980) showed that the HL-60 cell line, which was allegedly derived from a patient with APL, could be induced to undergo granulocytic differentiation by micromolar concentrations of ATRA. One year later, the same group of investigators showed that ATRA causes primary APL cells to differentiate in vitro. Although an obvious step forward, the clinical trial with ATRA did not immediately follow and it was only in 1987 that Huang and colleagues reported the successful use of ATRA to induce complete remission (CR) in patients with APL, without an apparent increase in the severity of the haemorrhagic diathesis observed at presentation (Huang et al, 1987). Incorporation of chemotherapy and up-front use of ATRA in combination with arsenic trioxide (ATO) lead to CR rates in excess of $93 \%$ with patients achieving 5 -year overall survival rates approaching $100 \%$. A recent phase 3 trial showed that combination treatment of ATRA and ATO without chemotherapy leads to $\mathrm{CR}$ and an extended overall survival compared with the standard ATRA and chemotherapy treatment $(P=0.02)$ in patients with low-risk APL (Lo-Coco et al, 2013). This success of ATRA in APL shows that the concept of differentiation therapy can be an effective strategy in leukaemia and provided a rationale for its use in the treatment of other cancers. The discovery that the $t(15 ; 17)$ translocation, the cytogenetic marker for APL involves the RARA gene on chromosome 17q21, reaffirmed the importance of ATRA and RAR $\alpha$ in myelomonocytic differentiation and paved the way for the understanding of the molecular mechanisms underlying the disease pathogenesis as well as this remarkable response to ATRA (Sirulnik et al, 2003).

The most common RARA translocation partner is the $P M L$ gene. Fusion of PML sequences to the B-F regions of RARA increases the affinity of the fusion receptor for co-repressors so that higher (pharmacological) levels of ATRA are required to induce its dissociation. Consistent with these findings, ability of PML/RAR $\alpha$ to block differentiation of transfected myeloid cells depends on an intact N-CoR-binding region in the RAR moiety of the fusion protein (Racanicchi et al, 2005).

\section{ATRA IN NON-APL AML AND OTHER MALIGNANCIES}

The seemingly selective effectiveness of ATRA in APL poses an important question of whether the presence of the PML/RAR $\alpha$ rearrangements in this malignancy renders it uniquely susceptible to ATRA therapy. One argument against such a view is that ATRA effectiveness in AML has been observed in cells that lack PML/ RAR $\alpha$, as HL-60 cells, that can be induced to differentiate into mature granulocytes (Breitman et al, 1980). Furthermore, clinical studies with ATRA in previously untreated older patients with AML yielded some encouraging results and various clinical trials throughout the World have shown effectiveness of ATRA when used in combination with other agents such as conventional chemotherapy. Di Febo et al (2007) found that treatment with low-dose cytosine arabinoside (LDAC) and ATRA lead to an improvement of the time to treatment failure rate and longer 
survival in patients with AML older than 60 years in comparison with LDAC alone (Di Febo et al, 2007). Similarly, Schlenk et al (2009) reported that in elderly patients with AML the genotype 'mutant NPM1 without FLT3-ITD' appears to be a predictive marker for response to ATRA given in addition to intensive chemotherapy. Venditti et al (1995) also observed that the combined use of ATRA and LDAC was effective for inducing $\mathrm{CR}$ in patients with 'poor prognosis' AML, especially in patients with $<50 \%$ bone marrow infiltration (Venditti et al, 1995). However, other phase 2 studies showed no or non-significant effects (Milligan et al, 2006; Burnett et al, 2007). Overall, these studies show that the efficacy achieved so far with ATRA in patients with non-APL AML is limited and there is much room for improvement.

Use of ATRA in anticancer therapy. Given the specific role of ATRA in cell proliferation and differentiation, its potential effectiveness to treat human cancers has captivated researchers for decades. Effects of ATRA on tumour development are extensive and include differentiation and apoptosis induction, as well as tumour prevention (Altucci and Gronemeyer, 2001). For example, the ATRA derivative 13-cis-retinoic acid (13-cis-RA) is successfully used in the treatment of children with high-risk neuroblastoma, reducing the risk of recurrence after high-dose chemotherapy and stem cell transplantation and increasing long-term survival (Matthay et al, 2009). Nevertheless, so far ATRA is only routinely and effectively used to treat APL and precancerous lesions such as leukoplakia, actinic keratosis, and cervical dysplasia.

Consistent with the important role of ATRA signalling in cellular homeostasis and potential tumorigenic consequences of deregulation in this signalling pathway are reports by Losson and colleagues showing that loss of Tif1 alpha renders RAR $\alpha$ oncogenic in the liver (Khetchoumian et al, 2008).

In recent years, ATRA has been studied in combinations with other drugs in a variety of cancers and precancerous conditions. Currently, several clinical trials using ATRA as part of combination therapies are ongoing. Tamoxifen, an antioestrogen with antiproliferative effects, is more effective against breast carcinoma and hepatoma cells when combined with retinoids. The combination of dexamethasone and ATRA has also been shown to inhibit proliferation and induce differentiation in human osteosarcoma cells. It has also been observed that Vitamin D3 analogues combined with ATRA can reduce the breast tumour mass in nude mice and inhibit cell growth and induce apoptosis in lung, prostate, breast, and ovarian cancer cells in vitro. In addition, the use of retinoids against secondary liver cancer, aerodigestive malignancies and secondary breast cancer has shown promising results (Clarke et al, 2004).

All-trans-retinoic acid has also been evaluated in combination with G-CSF and GM-CSF. All-trans-retinoic acid and G/GM-CSF co-operate to promote myelomonocytic differentiation in murine pluripotent myeloid progenitor cells, different AML cell lines, as well as primary cells from patients with myeloid leukaemia (Matsui et al, 2005). The ligand-dependent activities of endogenous and transiently expressed RAR $\alpha$ isoforms are enhanced by G/GM-CSF and correlate with an increased expression of ATRA inducible genes as well as leukaemic cell differentiation (Glasow et al, 2005). These data suggest that the manipulation with small molecules of the signalling pathways downstream from the G/GM-CSF receptors in AML cells may be an effective strategy to maximise the effects of ATRA in AML therapy.

Another way to enhance ATRA-stimulated differentiation is the use of inhibitors of inosine $5^{\prime}$-monophosphate dehydrogenase (IMPDH). Inosine $5^{\prime}$-monophosphate dehydrogenase has an important role in the maintenance of homeostasis of guanosine nucleotides in the cell. Higher levels of guanosine nucleotides as well as IMPDH can be found in many cell types, including myeloid cells, and depletion of guanosine nucleotides are known to accompany their differentiation.

The use of telomerase inhibitors has also been proposed to improve ATRA responsiveness. Recent studies suggest that one of the effects of ATRA and ATO in APL is due to a synergy at the level of inhibition of telomerase activity. Stem cells exhibit higher telomerase activity compared with their normal counterparts and downregulation of the hTERT gene and a concomitant reduction in telomerase activity is an early event following induction of differentiation by ATRA. Finally, combination of ATRA with different interferons (IFNs) have been shown to enhance effects of both drugs and lead to growth suppression and cell death in many tumour cell lines via a set of genes associated with retinoid-IFNinduced mortality' (GRIMs) (Hofmann et al, 1998).

Many mutations involving genes that mediate signalling pathways have a crucial role in tumorigenesis. These genes contribute to cell proliferation (e.g., FLT3, c-KIT, RAS, and PTPN11), are involved in differentiation (e.g., AML1 and CEBPA), or are implicated in cell-cycle regulation or apoptosis (e.g., P53 and NPM1). Despite them being ideal partners for ATRA, only a few treatments combining ATRA with inhibitors of signal transduction pathways have been described to date. In one study, it has been observed that the inhibition of the mitogen-activated protein kinase (MAPK) pathway sensitises AML cells with constitutive MAPK activation to retinoid- and chemotherapy-induced apoptosis (Milella et al, 2001). It has been observed in another study that RAD001 (everolimus), an inhibitor of mammalian target of rapamycin complex 1 (mTORC1), is able to reduce the stem cell potential and enhance the ATRA-induced growth arrest and differentiation of AML cells. Furthermore, RAD001 improves the ability of ATRA to inhibit the proliferation of HL-60 cells growing as tumour xenografts in immune-deficient nude mice (Nishioka et al, 2009). Inhibitors of the glycogen synthase kinase 3 (GSK3) have also been shown to inhibit growth and drive differentiation of a variety of leukaemic cells in combination with ATRA. GSK3b phosphorylates $\operatorname{RAR} \alpha$ on multiple residues reducing its transcriptional activity. The inhibition of GSK3b prevents RAR $\alpha$ phosphorylation and its degradation by the proteasome leading to increased levels of RAR $\alpha$ in the cell. So far only the FMS-like tyrosine kinase 3 (FLT3) inhibitor midostaurin- and cladribine-based induction chemotherapy (CLAG) have been studied in a clinical trial in combination with ATRA in relapsed or refractory AML.

Another way to improve responsiveness to ATRA is to increase its intracellular levels by inhibiting the activity of the aldoketoreductase AKR1C3. AKR1C3 is an NADPD-dependent oxidoreductase that has a role in the metabolism of retinoids and can therefore control the level of ATRA in the cell. Acute myeloid leukaemia cells have been shown to express AKR1C at high levels and inhibition of AKR1C leads to increased responsiveness of AML cells to ATRA (Brown and Hughes, 2012).

Epigenetic regulation of RARA and combinatorial use of Epi-drugs and ATRA. The promoter of the RARA isoform RARA2, but not RARA1, is hypermethylated in AML cell lines, and induction of differentiation and expression of RARA2 can be stimulated with DNA demethylating agents and ATRA treatment. Consistent with what has been observed in cell lines, levels of RAR $\alpha 2$ expression are also markedly reduced in AML patient samples compared with normal myeloid cells expressing CD33 or CD34. However, in contrast to AML cell lines and normal myeloid cells, expression of the RAR $\alpha 1$ isoform is also significantly reduced in primary AML cells. Moreover, both RARA1 and RARA2 promoters (Glasow et al, 2008) are unmethylated in AML patient samples, while histones associated with the RARA2 promoter have decreased levels of overall acetylation of histone $\mathrm{H} 3$ and decreased levels of dimethylation of histone $\mathrm{H} 3$ lysine 4 . Both are heterochromatin markers associated with reduced gene expression indicating that 
A

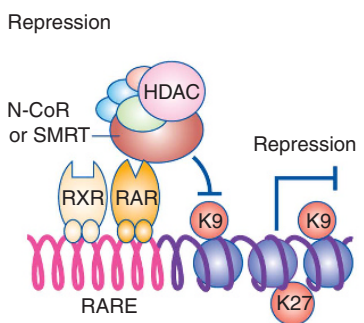

B

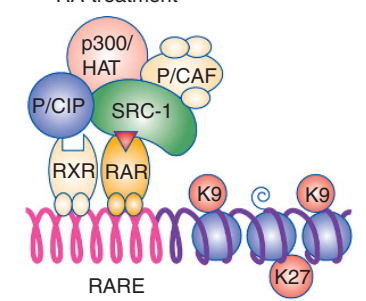

C

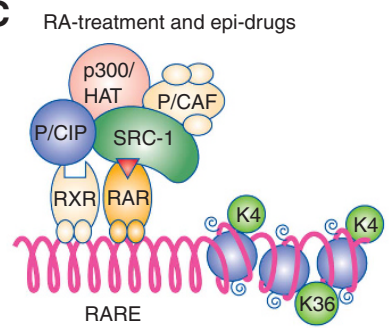

epi-drugs
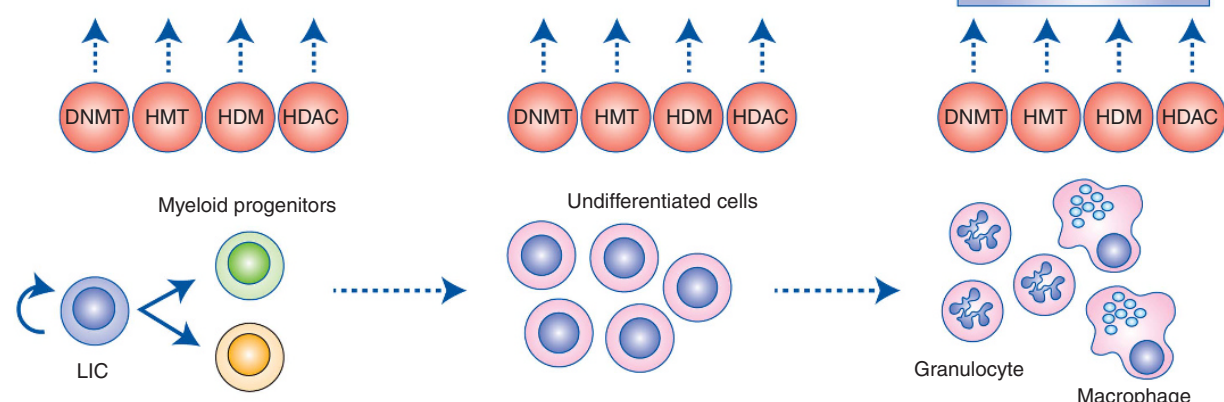

Undifferentiated cells
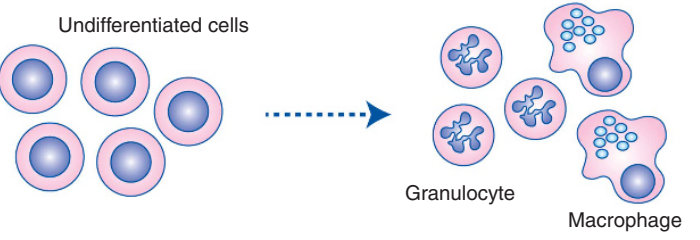

Epigenetic marks:

Repression

O DNA CpG-methylation K9 H3K9 me3 K27) H3K27 me3

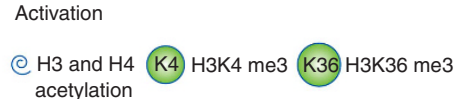

Figure 3. Mechanism of action of ATRA/epi-drug combinations. (A) Retinoic acid receptors, not bound by ligand, complex with co-repressors and HDACs to actively inhibit gene expression. Cancerous cells such as leukaemia initiating cells (LICs) are frequently characterised by activating mutations or an overexpression of epigenetic regulators (DNMTs, HMTs, HDMs, and HDACs) causing a false repression of tumour suppressor genes or genes involved in differentiation signalling pathways. (B) ATRA treatment alone is not sufficient to unblock RAR target genes do to epigenetic dysregulation. (C) Treatment of cancer cells by epigenetic (epi)-drugs in combination with ATRA unblocks the transcriptional repressive state of RAR target genes and allows the activation gene such as those involved in differentiation signalling pathways.

epigenetically driven decrease in RARA expression may be a factor in the pathogenesis of AML and contribute to the differentiation blockade.

Many mutations contribute to tumorigenesis by causing transcriptional deregulation through epigenetic modifications resulting in aberrant proliferation, impaired differentiation, and impaired apoptosis. These epigenetic modifications include changes in DNA methylation and histone modifications such as acetylation and methylation. Some studies have indicated that the combination of ATRA and epigenetic (epi)-drugs such as DNA methyltransferase inhibitors (DNMTi) or histone deacetylase inhibitors (HDACi) has higher therapeutic value in AML disease and potentially in other malignancies (Figure 3). Aberrant $\mathrm{CpG}$ island methylation in promoter regions in genes coding for tumour suppressors is a well-described phenomenon. CpG island methylation is associated with transcriptional silencing, involving the recruitment of methyl-binding proteins and HDACs to regions close to transcriptional initiation sites (Garcia-Manero et al, 2009).

Over the last years, several epigenetic drugs have been approved or are in clinical development (Dawson and Kouzarides, 2012). Two of the first epigenetic drugs used in AML were the nucleoside analogues 5-azacytidine and 5-aza-2'deoxycytidine. These DNMTi are incorporated into the DNA, where they form a covalent complex with the DNMT enzyme. This results in the degradation of the enzyme and a progressive loss of DNMT activity and the aberrant CpG island hypermethylation in cancer cells. Histone deacetylase inhibitors have been demonstrated to suppress tumours with $\mathrm{t}(15 ; 17)$ translocations in nude mice, and induce remission in transgenic mouse models of ATRA-resistant APL (He et al, 2001). Histone deacetylase inhibitors also induce differentiation and apoptosis of $\mathrm{t}(8 ; 21)$ leukaemia cell lines and primary leukaemia blasts (Wang et al, 1999). Although HDACi have been used both alone and in combination with ATRA in patients with
AML (including APL) the results were of limited value. One reason could be that these agents fail to clear leukaemia-initiating cells (LICs) (Leiva et $a l, 2012$ ). An interesting new approach to specifically target the repressive PML-RAR $\alpha$-HDAC complex in APL has been recently published by Bellis et al (2014). Using a hybrid molecule of ATRA with a 2-aminoanilide tail of the HDAC inhibitor MS-275 (MC2392), they were able to selectively induce apoptosis in cells carrying the PML-RAR $\alpha$ fusion gene.

We have recently demonstrated a critical role for the histone $\mathrm{H} 3$ lysine $4 \mathrm{mono} / \mathrm{di}(\mathrm{H} 3 \mathrm{~K} 4 \mathrm{me} 1 / \mathrm{me} 2)$ demethylase LSD1 (KDM1A, AOF2) as a negative regulator of the ATRA-mediated myeloid differentiation pathway (Schenk et al, 2012). LSD1 is highly expressed in AML patients (www.proteinatlas.org) and its overexpression has been correlated with impaired survival in various other tumours (Hayami et al, 2011). Overexpression of LSD1 in AML is associated with alterations in the histone methylation status and repression of ATRA-responsive genes. We showed that combination treatments with inhibitors of LSD1 and ATRA can dramatically enhance the pro-differentiative effects of ATRA in vitro and in vivo. Co-treatment with ATRA and tranylcypromine (TCP) greatly increased the fraction of $\mathrm{CD}_{11 \mathrm{~b}}{ }^{+}$cells by 21 -fold and 16fold in HL-60 and TEX cells, respectively. Mirroring the results in cell lines, co-treatment with ATRA and TCP increased the fraction of $\mathrm{CD} 11 \mathrm{~b}^{+}$cells in primary AML patient samples by a factor up to 11-fold and induced morphological changes, including formation of cytoplasmic neutrophil granules, consistent with differentiation of patient blasts into polymorphonuclear leukocytes. The treatment also lead to diminished clonogenic capacity of AML cells in vitro and engraftment of primary AML cells from patients in vivo in NOD.SCID gamma (NSG) mice, suggesting that LSD1i/ATRA combinations may also target LICs. We showed that these effects are at least in part due to gene-specific increases in $\mathrm{H} 3 \mathrm{~K} 4 \mathrm{me} 2$ methylation leading to expression of a subset of genes associated 
with the myelomonocytic differentiation pathway. This work shows that LSD1 is an important therapeutic target and highlights the potential for drugs targeting aberrant epigenetics in combination with retinoids (Figure 3).

Several major pharmaceutical companies are currently developing selective LSD1 inhibitors with micromolar potency and activity against a panel of cancer cell lines (Cain, 2012). Phase I trials evaluating treatment with LDS1 inhibitors in several hematologic malignancies are expected to begin this year.

\section{FUTURE OF ATRA IN CANCER THERAPY?}

Despite important advances in cancer therapeutics over the past few decades, treatment failure, relapse. and mortality rates in the majority of malignant diseases, including non-APL AML, remain unacceptably high. It is reasonable to predict that cure rates will not improve significantly unless alternative treatment modalities to conventional chemotherapy regimens and bone marrow transplantation are developed. Treatment of AML with targeted therapies is particularly promising due to the fact that AMLs have far fewer mutations than most other adult cancers, with an average of only 13 mutations (Mardis et al, 2009; Cancer Genome Atlas Research Network, 2013). The availability of relatively inexpensive new techniques with which cancer-causing mutations in individual patients can be analysed, open the possibility for personalised anticancer therapies. Therefore, the goal has to be the development of a toolbox of drugs that can be used in a personalised manner.

All-trans-retinoic acid is a promising compound for treatment of a variety of cancers because of its specific effects on cell proliferation, differentiation, and apoptosis, as well as its low toxicity. Retinoic acid receptors are not mutated in cancer cells, so ATRA could potentially exert its anticancer effects in many malignancies. Nevertheless, to unleash the therapeutic potentials of ATRA, many studies emphasise the need for a better understanding of the mechanisms that block ATRA signalling and ATRA regulated gene expression in cancer, including AML.

It is evident that combinatorial therapies targeting multiple gene silencing mechanisms may be the most effective strategy in reactivating ATRA-sensitive gene expression and differentiation of AML cells, as well as mediating anticancer activities of ATRA in general. The identification of classes of proteins that control gene expression via histone and DNA modifications is driving the development of new therapeutic agents, the so-called 'epi-drugs', that alter chromatin structure. These epigenetic modifying drugs have been shown to be only partly effective against different cancers when used alone. In the context of non-APL AML, epi-drugs such as LSD1 inhibitors are most effective when combined with ATRA and vice versa. Further explorations of such combinatorial strategies may very well unravel multiple novel pathways/targets to unlock and rejuvenate the potential of ATRA in anticancer therapy.

\section{REFERENCES}

Al Tanoury Z, Piskunov A, Rochette-Egly C (2013) Vitamin A and retinoid signaling: genomic and nongenomic effects. J Lipid Res 54(7): $1761-1775$

Altucci L, Gronemeyer H (2001) The promise of retinoids to fight against cancer. Nat Rev Cancer 1(3): 181-193.

Bellis FD, Carafa V, Conte M, Rotili D, Petraglia F, Matarese F, Francoijs KJ, Ablain J, Valente S, Castellano R, Goubard A, Collette Y, Mandoli A, Martens JHA, The Hd, Nebbioso A, Mai A, Stunnenberg HG, Altucci L (2014) Context-selective death of acute myeloid leukemia cells triggered by the novel hybrid retinoid-HDAC inhibitor MC2392. Cancer Res 74(8): 2328-2339.

Breitman T, Selonick S, Collins S (1980) Induction of differentiation of the human promyelocytic leukemia cell line (HL-60) by retinoic acid. Proc Natl Acad Sci USA 77(5): 2936-2940.
Brown G, Hughes P (2012) Retinoid differentiation therapy for common types of acute myeloid leukemia. Leuk Res Treatment 2012: 939021.

Burnett A, Milligan D, Prentice A, Goldstone A, McMullin M, Hills R, Wheatley K (2007) A comparison of low-dose cytarabine and hydroxyurea with or without all-trans retinoic acid for acute myeloid leukemia and high-risk myelodysplastic syndrome in patients not considered fit for intensive treatment. Cancer 109(6): 1114-1124.

Cain C (2012) AML takes LSD1. Sci Business eXchange 5(14): 1-3.

Cancer Genome Atlas Research Network (2013) Genomic and epigenomic landscapes of adult de novo acute myeloid leukemia. $N$ Engl J Med 368(22): 2059-2074.

Clarke N, Germain P, Altucci L, Gronemeyer H (2004) Retinoids: potential in cancer prevention and therapy. Expert Rev Mol Med 6(25): 1-23.

Dawson M, Kouzarides T (2012) Cancer epigenetics: from mechanism to therapy. Cell 150(1): 12-27.

Di Febo A, Laurenti L, Falcucci P, Tosti ME, Fianchi L, Pagano L, Leone G (2007) All-trans retinoic acid in association with low dose cytosine arabinoside in the treatment of acute myeoid leukemia in elderly patients. Am J Ther 14(4): 351-355.

Garcia-Manero G, Yang H, Kuang SQ, O’Brien S, Thomas D, Kantarjian H (2009) Epigenetics of acute lymphocytic leukemia. Semin Hematol 46(1): 24-32.

Giannì M, Bauer A, Garattini E, Chambon P, Rochette-Egly C (2002) Phosphorylation by $\mathrm{p} 38 \mathrm{MAPK}$ and recruitment of SUG- 1 are required for RA-induced RAR gamma degradation and transactivation. EMBO J 21(14): 3760-3769.

Glasow A, Barrett A, Petrie K, Gupta R, Boix-Chornet M, Zhou DC, Grimwade D, Gallagher R, von Lindern M, Waxman S, Enver T, Hildebrandt G, Zelent A (2008) DNA methylation-independent loss of RARA gene expression in acute myeloid leukemia. Blood 111(4): 2374-2377.

Glasow A, Prodromou N, Xu K, von Lindern M, Zelent A (2005) Retinoids and myelomonocytic growth factors cooperatively activate RARA and induce human myeloid leukemia cell differentiation via MAP kinase pathways. Blood 105(1): 341-349.

Glass C, Rosenfeld M (2000) The coregulator exchange in transcriptional functions of nuclear receptors. Genes Dev 14(2): 121-141.

Hayami S, Kelly JD, Cho HS, Yoshimatsu M, Unoki M, Tsunoda T, Field HI, Neal DE, Yamaue H, Ponder BA, Nakamura Y, Hamamoto R (2011) Overexpression of LSD1 contributes to human carcinogenesis through chromatin regulation in various cancers. Int J Cancer 128(3): 574-586.

He LZ, Tolentino T, Grayson P, Zhong S, Warrell Jr. RP, Rifkind RA, Marks PA, Richon VM, Pandolfi PP (2001) Histone deacetylase inhibitors induce remission in transgenic models of therapy-resistant acute promyelocytic leukemia. J Clin Investig 108(9): 1321-1330.

Hofmann E, Boyanapalli M, Lindner D, Weihua X, Hassel B, Jagus R, Gutierrez P, Kalvakolanu D, Hofman E (1998) Thioredoxin reductase mediates cell death effects of the combination of beta interferon and retinoic acid. Mol Cell Biol 18(11): 6493-6504.

Huang ME, Ye YC, Zhao L (1987) [Treatment of acute promyelocytic leukemia by retinoic acid with or without low dose cytosine arabinoside: report of 4 cases]. Zhonghua Nei Ke Za Zhi 26(6): 330-332380.

Khetchoumian K, Teletin M, Tisserand J, Herquel B, Ouararhni K, Losson R (2008) Trim24 (Tif1 alpha): an essential 'brake' for retinoic acid-induced transcription to prevent liver cancer. Cell Cycle (Georgetown, Tex) 7(23): 3647-3652.

Leid M, Kastner P, Chambon P (1992) Multiplicity generates diversity in the retinoic acid signalling pathways. Trends Biochem Sci 17: 427-433.

Leiva M, Moretti S, Soilihi H, Pallavicini I, Peres L, Mercurio C, Dal Zuffo R, Minucci S, de Thé H (2012) Valproic acid induces differentiation and transient tumor regression, but spares leukemia-initiating activity in mouse models of APL. Leukemia 26(7): 1630-1637.

Lo-Coco F, Avvisati G, Vignetti M, Thiede C, Orlando S, Iacobelli S, Ferrara F, Fazi P, Cicconi L, Di Bona E, Specchia G, Sica S, Divona M, Levis A, Fiedler W, Cerqui E, Breccia M, Fioritoni G, Salih H, Cazzola M, Melillo L, Carella A, Brandts C, Morra E, von Lilienfeld-Toal M, Hertenstein B, Wattad M, Lübbert M, Hänel M, Schmitz N, Link H, Kropp M, Rambaldi A, La Nasa G, Luppi M, Ciceri F, Finizio O, Venditti A, Fabbiano F, Döhner K, Sauer M, Ganser A, Amadori S, Mandelli F, Döhner H, Ehninger G, Schlenk R, Platzbecker U. Gruppo Italiano Malattie Ematologiche dA, German-Austrian Acute Myeloid Leukemia Study Group, Study Alliance Leukaemia (2013) Retinoic acid and arsenic trioxide for acute promyelocytic leukemia. N Engl J Med 369(2): 111-121.

Mardis E, Ding L, Dooling D, Larson D, McLellan M, Chen K, Koboldt D, Fulton R, Delehaunty K, McGrath S, Fulton L, Locke D, Magrini V, Abbott R, Vickery T, Reed J, Robinson J, Wylie T, Smith S, Carmichael L, Eldred J, 
Harris C, Walker J, Peck J, Du F, Dukes A, Sanderson G, Brummett A, Clark E, McMichael J, Meyer R, Schindler J, Pohl C, Wallis J, Shi X, Lin L, Schmidt H, Tang Y, Haipek C, Wiechert M, Ivy J, Kalicki J, Elliott G, Ries R, Payton J, Westervelt P, Tomasson M, Watson M, Baty J, Heath S, Shannon W, Nagarajan R, Link D, Walter M, Graubert T, DiPersio J, Wilson R, Ley T (2009) Recurring mutations found by sequencing an acute myeloid leukemia genome. $N$ Engl J Med 361(11): 1058-1066.

Matsui W, Smith B, Vala M, Beal N, Huff C, Diehl L, Jones R (2005) Requirement for myeloid growth factors in the differentiation of acute promyelocytic leukaemia. Br J Haematol 128(6): 853-862.

Matthay KK, Reynolds CP, Seeger RC, Shimada H, Adkins ES, Haas-Kogan D, Gerbing RB, London WB, Villablanca JG (2009) Long-term results for children with high-risk neuroblastoma treated on a randomized trial of myeloablative therapy followed by 13-cis-retinoic acid: a children's oncology group study. J Clin Oncol 27(7): 1007-1013.

Milella M, Kornblau SM, Estrov Z, Carter BZ, Lapillonne H, Harris D, Konopleva M, Zhao S, Estey E, Andreeff M (2001) Therapeutic targeting of the MEK/MAPK signal transduction module in acute myeloid leukemia. J Clin Investig 108(6): 851-859.

Milligan D, Wheatley K, Littlewood T, Craig J, Burnett A. Group NHOCS (2006) Fludarabine and cytosine are less effective than standard ADE chemotherapy in high-risk acute myeloid leukemia, and addition of G-CSF and ATRA are not beneficial: results of the MRC AML-HR randomized trial. Blood 107(12): 4614-4622.

Nishioka C, Ikezoe T, Yang J, Gery S, Koeffler HP, Yokoyama A (2009) Inhibition of mammalian target of rapamycin signaling potentiates the effects of all-trans retinoic acid to induce growth arrest and differentiation of human acute myelogenous leukemia cells. Int J Cancer 125(7): 1710-1720.

Racanicchi S, Maccherani C, Liberatore C, Billi M, Gelmetti V, Panigada M, Rizzo G, Nervi C, Grignani F (2005) Targeting fusion protein/corepressor contact restores differentiation response in leukemia cells. EMBO J 24(6): 1232-1242.

Schenk T, Chen WC, Gollner S, Howell L, Jin L, Hebestreit K, Klein HU, Popescu AC, Burnett A, Mills K, Casero Jr. RA, Marton L, Woster P, Minden MD, Dugas M, Wang JC, Dick JE, Muller-Tidow C, Petrie K, Zelent A (2012) Inhibition of the LSD1 (KDM1A) demethylase reactivates the all-trans-retinoic acid differentiation pathway in acute myeloid leukemia. Nat Med 18(4): 605-611.

Schlenk R, Döhner K, Kneba M, Götze K, Hartmann F, Del Valle F, Kirchen H, Koller E, Fischer J, Bullinger L, Habdank M, Späth D, Groner S, Krebs B, Kayser S, Corbacioglu A, Anhalt A, Benner A, Fröhling S, Döhner H. German-Austrian AMLSG (2009) Gene mutations and response to treatment with all-trans retinoic acid in elderly patients with acute myeloid leukemia. Results from the AMLSG Trial AML HD98B. Haematologica 94(1): 54-60.

Sirulnik A, Melnick A, Zelent A, Licht J (2003) Molecular pathogenesis of acute promyelocytic leukaemia and APL variants. Best Pract Res Clin Haematol 16(3): 387-408.

Venditti A, Stasi R, Del Poeta G, Buccisano F, Aronica G, Bruno A, Pisani F, Caravita T, Masi M, Tribalto M (1995) All-trans retinoic acid and low-dose cytosine arabinoside for the treatment of 'poor prognosis' acute myeloid leukemia. Leukemia 9(7): 1121-1125.

Wang J, Saunthararajah Y, Redner RL, Liu JM (1999) Inhibitors of histone deacetylase relieve ETO-mediated repression and induce differentiation of AML1-ETO leukemia cells. Cancer Res 59(12): 2766-2769.

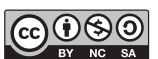

This work is licensed under the Creative Commons Attribution-NonCommercial-Share Alike 3.0 Unported License. To view a copy of this license, visit http://creativecommons. org/licenses/by-nc-sa/3.0/ 